fingers was captured by a sculpture (fig 1). A useless armamentarium of crude instruments was presented in theatre (fig 2). Her fingers were freed when my variable speed Hobbydrill was used with its cutting attachment; the blade of tissue forceps was interposed to protect the tissues. This equipment helped save the child's fingers. She was allowed home the next day, and there was no sign of the injury at follow up six weeks later.

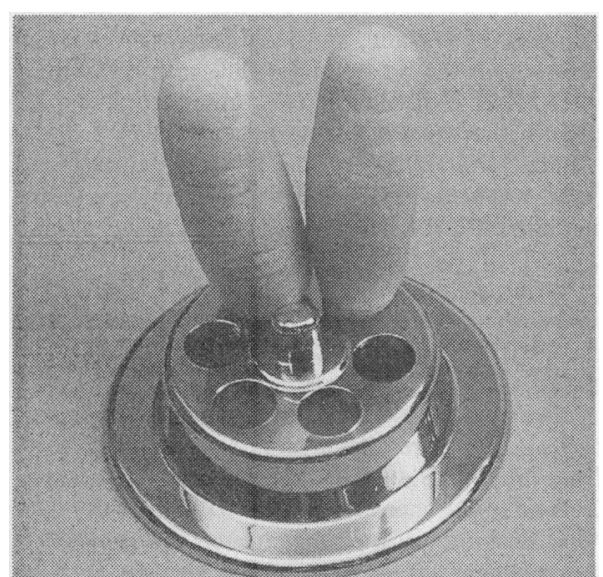

FIG 1-Sculpture of fingers caught in plughole

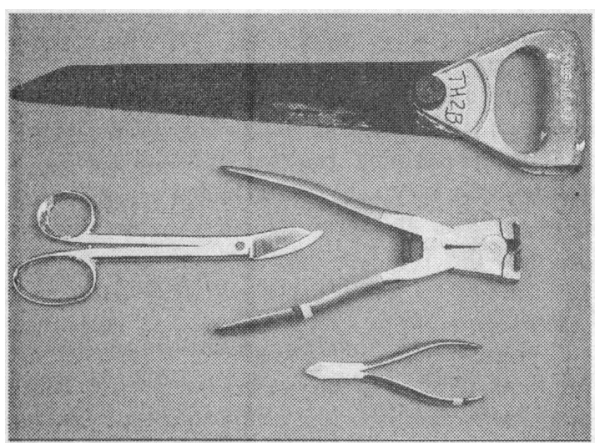

FIG 2-Useless instruments in theatre

The drill was in the hospital only because I intended to engrave the neonatal stethoscopes. It is an adaptable, controllable, and precision piece of equipment and would be a worthwhile investment (of about $£ 40$ ) for hospitals until manufacturers change the design of their waste outlets.

C A HAJIVASSILIOU

Royal Hospital for Sick Children,

Glasgow G3 8S]

1 Esberger DA. Photo finish. BMf 1993;307:1634. (18-25 December.)

2 Crowder S, Mitra S. Photo finish. BMf 1993;307:1634. (18-25 December.)

\section{Access to cardiac catheterisation}

\section{Influenced by deprivation, not sex}

EDrTor,-F Kee and colleagues have added to the continuing controversy regarding fair access to cardiological services. ${ }^{1}$ They found that the rate of cardiac catheterisation was significantly lower in women than men and noted no influence of social background. This is in contrast to our findings. ${ }^{2}$ We have updated our results and continue to find a strong influence of social deprivation on the uptake of both cardiac catheterisation and coronary bypass surgery, particularly in women (submitted for publication), but no significant sex bias in cardiac catheterisation based on patients discharged from hospital with coronary heart disease.

The following may explain the differing findings. We restricted our analysis to patients aged 35-64 since we thought that they were most likely to be affected by non-clinical variables: younger patients would almost certainly be investigated, irrespective of their social status, and older patients would be presented for investigation because of symptoms that could not reasonably be ignored on demographic grounds. The rates of cardiac catheterisation in our population were roughly four times higher than those in the population studied by Kee and colleagues. Our patients were allocated to eight groups according to their deprivation score; the groups were not equal in size but had comparable mixes of deprivation and affluence. In contrast, Kee and colleagues' patients were divided into fifths. The larger numbers of investigations and social groups in our study may have enhanced our ability to detect an influence of social deprivation. Furthermore, our higher rates of catheterisation may have allowed more liberal and subjective criteria to influence the decision to investigate.

These differences reinforce concerns about different patterns of investigation and treatment of patients with coronary heart disease. ${ }^{34}$

IAIN N FINDLAY

Royal Alexandra Hospital,

Paisley PA2 9PN

DAVID CUNNINGHAM

HENRY J DARGI

\section{Western Infirmary,}

Glasgow G11 6NT

1 Kee F, Gaffney B, Currie S, O'Reilly D. Access to coronary catheterisation: fair shares for all. $B M$ f 1993;307:1305-7. (20 November.)

2 Findlay $\mathrm{IN}$, Dargie HJ, Dyke T. Coronary angiography in Glasgow: relation to coronary heart disease and social class. $B r$ Heart $f$ 1 $1991 ; 66: 70$.

3 Findlay IN, Cunningham D, Dargie HJ. The effect of cardiac catheterisation facilities on the rate of coronary bypass grafting in Scotland. Br Heart F 1992;68:69.

4 Clinical Standards Advisory Group. Access to and availability of coronary artery bypass grafting and coronary angioplasty. London: HMSO, 1991.

\section{Access to surgery linked to social class}

EDIroR,-F Kee and colleagues report the variation in rates of cardiac catheterisation among electoral wards in Northern Ireland and suggest that social deprivation has little influence on the rate once a proxy measure of clinical need has been taken into account. ${ }^{1}$ We examined the relation between social class and rates of coronary artery bypass grafting in 7735 men aged 40-59 at entry to the British regional heart study, a prospective investigation of cardiovascular disease in 24 town in England, Wales, and Scotland. Information on social class was based on occupation at entry to the study in 1978-80. Details of coronary artery bypass operations and major ischaemic heart disease events were obtained by annual review of the patients' records supplemented by tagging for fatal events at the NHS central register. ${ }^{2}$

By 1992, 91 men in the original cohort were reported to have undergone coronary artery bypass grafting. Forty $(1 \cdot 31 \%)$ of the men with a nonmanual occupation had undergone such grafting compared with $48(1.08 \%)$ with a manual occupation (odds ratio (non-manual:manual) 1.21 (95\% confidence interval 0.78 to 1.89 )). The higher rate of coronary artery bypass grafting in the non-manual group contrasts with the lower proportion of men in this group who had either evidence of ischaemic heart disease at entry to the study (odds ratio $0.65(0.57$ to 0.75$)$ ) or a major fatal or non-fatal ischaemic heart disease event during follow up (odds ratio $0.74(0.61$ to 0.88$)$ ).

These results suggest that social class differences in rates of coronary artery bypass grafting may not reflect clinical need, at least in this study population. The extent to which the imbalance observed reflects differences in rates of cardiac catheterisation and social class differences in acceptance rates for operation, possibly influenced by smoking ${ }^{3}$ and other clinical and social factors, requires further exploration.

RICHARD W MORRIS

Department of Public Health,

Royal Free Hospital School of Medicine,

London NW3 2PF

1 Kee F, Gaffney B, Currie S, O'Reilly D. Access to cardiac catheterisation: fair shares for all? $B M \mathcal{F}^{1}$ 1993;307:1305-7. (20 November.)

2 Walker M, Shaper AG. Follow-up of subjects for prospective studies in general practice. $\mathcal{F}$ R Coll Gen Pract 1984;34:365-70.

3 Underwood MJ, Bailey JS, Shiu M, Higgs R, Garfield J. Should smokers be offered coronary bypass surgery? $B M \mathcal{F}$ 1993;306: $1047-50$

\section{Home visits by general practitioners}

EDrTor,-Linda Beecham reports on the current state of negotiations about the reorganisation of out of hours emergency services by general practitioners. ${ }^{1}$ The accompanying photograph of a young general practitioner visiting an elderly woman at home is ironic, because those who argue in favour of emergency centres overlook two important facts.

The first fact is that the overwhelming majority of home visits are made to patients over 65 , who often have multiple diagnoses and are the least able to travel to emergency centres. I recently showed this in an audit of home visits in a semirural practice in Norfolk: 207 out of 265 visits were to patients over 65 , in whom the three commonest diagnoses were respiratory tract infections, dizziness, and joint pain. These are clearly the patients who are least likely to be able to travel to an emergency centre.

The second fact often overlooked is that many general practitioners already try, whenever possible, to see patients out of hours on surgery premises, so that notes are available and treatment facilities are to hand.

As with many other aspects of the new health service, we are being encouraged to reinvent the wheel for a vehicle whose main problem is shortage of fuel. We should not sanction a change in our conditions of service that will ultimately reduce the standard of care that we offer to some of our most vulnerable patients.

Coltishall

MICHAEL J NOBLE

Norfolk NR12 7DW

1 Beecham L. Home visits will fall with new GP scheme. BMI 1993;307:1375. (27 November.)

\section{Selective decontamination of the digestive tract}

EDrToR,-M J M Bonten and colleagues agree with us that meta-analysis helps to clarify the methodological quality and clinical consistency of published research. ${ }^{1}$ They also acknowledge the merits of our recent review of trials of selective decontamination of the digestive tract in making clear important differences in study design, population, and methods that may explain why results of different studies differ. ${ }^{2}$ They criticise us, however, for using the "number to be treated" as a measure of the effect of treatment. They argue that only trials in which the incidence of pneumonia was high showed a positive effect of selective decontamination. Such a statement does not help in understanding the difference between statistical significance, clinical relevance, and cost-benefits. Everybody running an intensive care unit has to appraise the scientific evidence before deciding 